\title{
Osteosarcoma arising from osteochondroma of the tibia: case report and cytogenetic findings
}

\author{
E.E. Engel ${ }^{1}$, M.H. Nogueira-Barbosa ${ }^{2}$, M.S. Brassesco ${ }^{3}$, G.E.B. Silva ${ }^{4}$, \\ E.T. Valera ${ }^{3}$, F.M. Peria ${ }^{5}$, T.C. Motta ${ }^{5}$ and L.G. Tone ${ }^{3}$
}

'Departamento de Biomecânica, Medicina e Reabilitação do Aparelho Locomotor, Faculdade de Medicina de Ribeirão Preto, Universidade de São Paulo, Ribeirão Preto, SP, Brasil

${ }^{2}$ Departamento de Medicina Interna, Faculdade de Medicina de Ribeirão Preto, Divisão de Diagnóstico por Imagem, Universidade de São Paulo,

Ribeirão Preto, SP, Brasil

${ }^{3}$ Departamento de Puericultura e Pediatria,

Faculdade de Medicina de Ribeirão Preto, Divisão de Oncologia Pediátrica, Universidade de São Paulo, Ribeirão Preto, SP, Brasil

${ }^{4}$ Departamento de Patologia, Faculdade de Medicina de Ribeirão Preto, Universidade de São Paulo, Ribeirão Preto, SP, Brasil

${ }^{5}$ Departamento de Medicina Interna,

Faculdade de Medicina de Ribeirão Preto, Divisão de Oncologia,

Universidade de São Paulo, Ribeirão Preto, SP, Brasil

Corresponding author: E.E. Engel

E-mail: engel@fmrp.usp.br

Genet. Mol. Res. 11 (1): 448-454 (2012)

Received February 6, 2011

Accepted November 26, 2011

Published March 1, 2012

DOI http://dx.doi.org/10.4238/2012.March.1.1

\begin{abstract}
Osteochondroma is a cartilage capped benign tumor developing mainly at the juxta-epiphyseal region of long bones. The rate of malignant transformation, mainly into chondrosarcoma, is estimated to be less than $1-3 \%$. Transformation into osteosarcoma is very rare and has been reported only thirteen times. There is little information on
\end{abstract}


treatment and outcome. We report the case of a secondary osteosarcoma arising in the left tibia of a 23-year-old male, 10 years after the initial diagnosis of osteochondroma and after two partial resections. Malignant transformation occurred at the stalk and not at the cartilage cap, as would normally be expected. Chromosome banding analysis revealed the karyotype: 46,XY, $t(3 ; 13)(\mathrm{q} 21 ; \mathrm{q} 34)$ [2]/46,XY [18]. Records from additional cases will help determine the parameters that define these rare secondary bone lesions.

Key words: Osteosarcoma; Bone neoplasms; Osteochondroma

\section{INTRODUCTION}

The World Health Organization (WHO) defines osteochondroma (OC) as a cartilagecapped bony projection arising on the external surface of bone containing a marrow cavity that is continuous with that of the underlying bone. OC may occur as sporadic solitary or multiple lesions, the latter characterizing hereditary osteochondromatosis (Khurana et al., 2002). OC has been shown to be a neoplastic disorder, documented by loss of heterozygosity at the EXT1 and EXT2 loci, DNA aneuploidy, and clonal karyotypic abnormalities (Bovée et al., 1999).

Malignant transformation of these benign tumors is very rare and is estimated to be less than 1 and $1-3 \%$ in solitary and multiple OC, respectively, while higher incidences have been considered to be bias in patient selection (Khurana et al., 2002). In general, transformation leads to chondrosarcomas in $94 \%$ of cases, affecting a population younger than that of primary chondrosarcomas (Lamovec et al., 1999; Bovée et al., 2002). Malignant transformation is suspected mainly if there is recent onset of pain without any adequate alternative explanation or continued growth of an OC after skeletal maturity. Plain radiographs may show irregular mineralization but the most reliable sign is the increased thickness of the cartilage cap, better seen by magnetic resonance imaging (MRI) (Lee et al., 2002). Ultrasound is also suitable for visualization of the cartilaginous cap thickness in superficial OCs (Lee et al., 2002). Cortical destruction and soft tissue mass may also be seen.

Secondary chondrosarcomas are generally low-grade tumors and have good prognosis (Lee et al., 2002). Malignant transformation of chondrosarcoma into osteosarcoma has seldom been reported in the literature. Lamovec et al. (1999) presented one case arising from a solitary OC of the fibula and reviewed the 10 previously described reports in the literature. Another case with no mutations in the EXT1 or EXT2 genes has also been reported (Bovée et al., 2002). More recently, Staals et al. (2007) published a 32-year survey from the Rizzoli Institute (Bologna, Italy), describing 12 cases of high-grade sarcomas arising from OCs from which nine were classified as osteosarcomas. Also, a single case of secondary osteosarcoma arising from an OC was described by Nishida et al. (2009). However, the patient had been a victim of the atomic bomb in Nagasaki, and the relationship between tumor development and radiation exposure still remains unclear. In total, thirteen of these unusual clinical situations were reported to date, reinforcing the rarity of this association.

In the present article, we report a case of a secondary osteosarcoma arising from an OC occurring in the left tibia, 10 years after the first resection. The article also reviews and discusses clinical and cytogenetic aspects associated with these entities. 


\section{Clinical history}

A 13-year-old male presented in 1997 complaints of pain and swelling on the left proximal tibia. A large-based sessile $\mathrm{OC}$ was seen by imaging examinations. As the lesion involved almost the entire circumference of the bone, the patient was submitted to a partial surgical resection.

Four years later, a local recurrence was noted. In the border of the previous resection, spiculous bone formations caused a prominence in the anteromedial aspect and limited knee motion in the popliteal region. Complaints were joint limitation and pain. Once more, the patient was submitted to partial resection of these prominences.

In 2009, the patient sought medical assistance, complaining of pain, and a bulge in the (postero-medial) aspect of the proximal tibia was observed. Imaging showed alterations suggesting malignant transformation. Histology of the resected bone revealed an osteosarcoma. Chemotherapy with methotrexate was not tolerated, and a six-cycle protocol with doxorubicin and cisplatin was administered. Currently, nine months after surgery, the patient is alive with no evidence of the disease.

\section{Imaging}

Initial X-ray findings, from 1997 and 2001, were consistent with the diagnosis of $\mathrm{OC}$ in the proximal region of the left tibia (Figure 1A and B), demonstrating the classic features of an exophytic and sessile expansive lesion. The CT and MRI performed in 2001 confirmed recurrence of the $\mathrm{OC}$ with characteristic imaging findings of this cartilaginous neoplasm (Figure 1C and D).

When the patient returned in 2009, MRI findings depicted a soft tissue mass with bone invasion in the posteromedial corner of the knee. This mass was predominantly characterized by high signal intensity but also showed internal areas of disorganized low signal on T2weighted MRI (Figure 1E and F).

\section{Histology}

The first specimen consisted of three roughly nodular pieces of bone tissue with a bosselated surface, measuring $2.8 \times 2.2 \times 1.5,2.6 \times 2.1 \times 1.0$ and $1.2 \times 1.0 \times 1.0 \mathrm{~cm}$. They had a cap of firm, gray, translucent cartilage, measuring between 5 and $12 \mathrm{~mm}$ in thickness. The cartilage cap merged into the underlying spongiosa with chondrocytes arranged according to an epiphyseal growth plate. A typical benign chondrocyte had a single small nucleus. The interior of the lesion was composed of normal spongy bone. These findings are compatible with a diagnosis of OC (Figure 2A). The second specimen showed the same findings, and the diagnosis was also OC.

The third specimen resected in 2009 showed a gross, irregularly shaped 10.0 x $6.5 \mathrm{x}$ $1.5 \mathrm{~cm}$ bony excrescence that projected from the surface of the tibial bone, and was partially covered by a bluish cartilaginous cap. Microscopic studies showed a dense cell mass with considerable hyperchromatism, nuclear atypia, and frequent mitoses. In some areas osteoid was formed by the tumor. Minimal chondroid differentiation was seen. The tumor was considered to be a low-grade osteosarcoma. There were few similar areas in the first lesion (Figure 2B and C). 
A

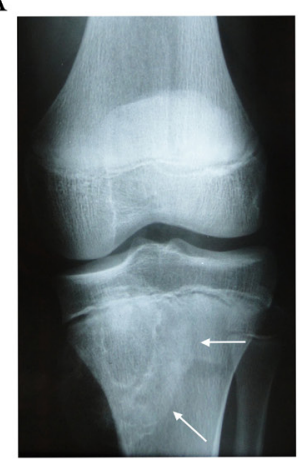

B

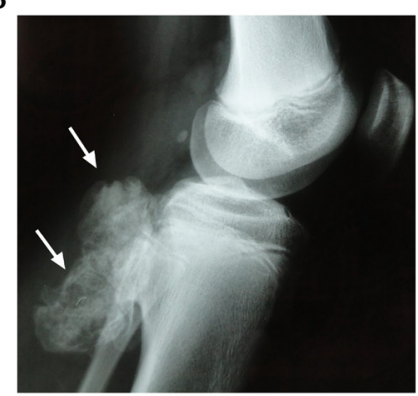

C

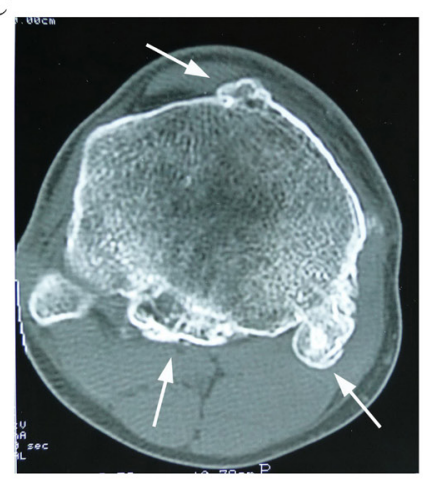

$\mathrm{D}$

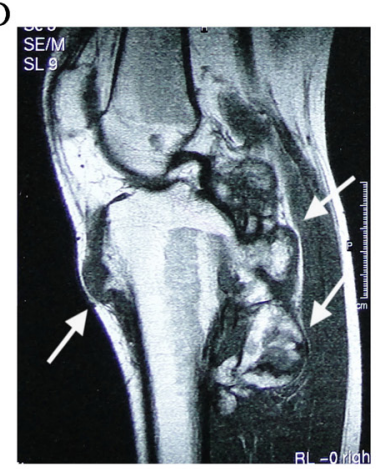

$\mathrm{E}$
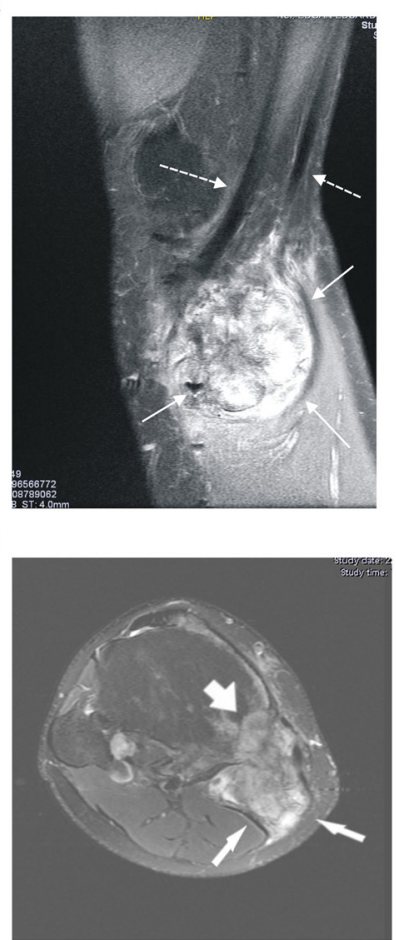

Figure 1. A. Evaluation of a palpable mass in the posterior region of the left knee in a 13-year-old boy at initial diagnosis in 1997, with an anteroposterior X-ray view. White arrows indicate the lateral border of the expansive metaphyseal lesion in the tibia. B. A lateral view X-ray obtained on the same occasion better demonstrates the exophytic behavior of the tumor (white arrows) and also the continuity of the tumor with tibia cortical and cancellous bone, a characteristic aspect of an osteochondroma. C. Evaluation of the same patient in 2001 with a CT axial image at the level of the tibial metaphysis demonstrated the circumferential growth of the osteochondroma in this case (arrows). D. A sagittal T1-weighted MRI obtained in the same year as in Figure 1C also confirmed tumor recurrence and showed characteristic imaging features of an osteochondroma (arrows). E. MRI performed in 2009 at the time of the last mass recurrence showing a heterogeneous soft tissue mass at the posteromedial corner of the left knee (white solid arrows) with central areas of low signal intensity on sagittal T2-weighted MRI. The flexor tendons at the posteromedial corner are pointed out with broken arrows). F. Axial T2-weighted MRI also showed the same features as noted in Figure 1E (white solid arrows) and demonstrated bone invasion (thick and short arrow). 
A

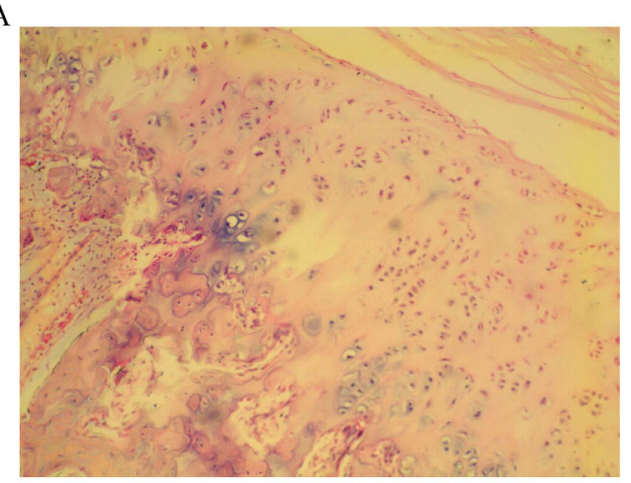

C

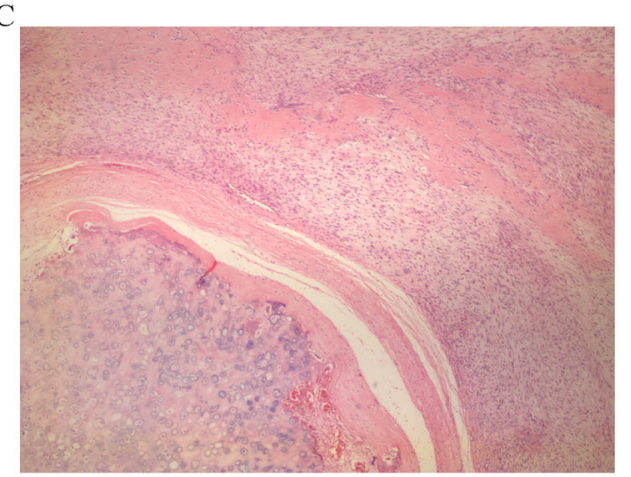

$\mathrm{B}$

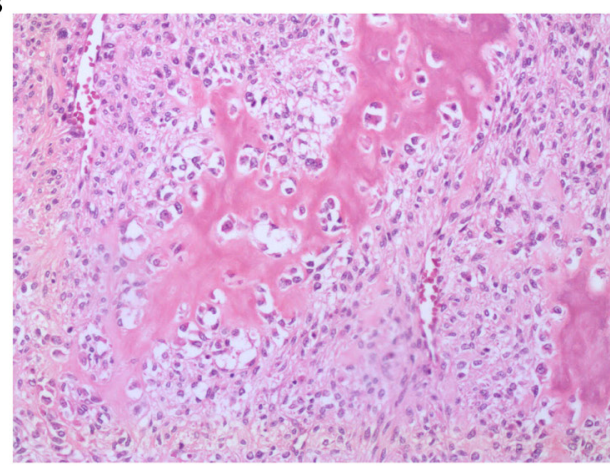

$\mathrm{D}$

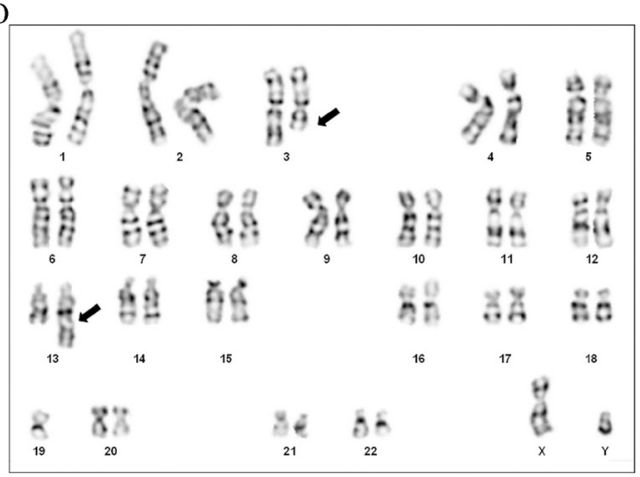

Figure 2. A. Microscopic appearance in the first lesion - osteochondroma. A perichondrium covers the cartilage cap. The cap merges into the underlying spongiosa, where the chondrocytes are arranged according to an epiphyseal growth plate. Endochondral ossification produces regular bony trabeculae with fat marrow (H\&E, 200X). B. Microscopic appearance in the third lesion showing spindle-shaped tumor cells with hyperchromatic, pleomorphic nuclei and some mitotic figures. Clear osteoid formation in the intercellular matrix is noted (H\&E, 400X). C. Microscopic appearance in the third lesion - osteosarcoma. The remaining osteochondroma is seen in the upper area and the sarcomatous area below (H\&E, 200X). D. GTG-banded metaphase showing a 46,XY, t(3;13)(q21;q34), -19 karyotype.

\section{Tumor culture and cytogenetic analysis}

A fresh osteosarcoma sample (adjacent to areas of tumor verified by frozen section), was aseptically collected in the operating room, and minced with scissors on a Petri dish. The tumor pieces were then disaggregated for $4 \mathrm{~h}$ on $0.5 \%$ collagenase type IV (Sigma Chemical Co., St. Louis, MO, USA) in F10 medium (Gibco BRL, Life Technologies, Carlsbad, CA, USA). Following the enzymatic treatment, the cells were centrifuged and the collagenase solution removed and replaced with medium supplemented with $10 \%$ fetal bovine serum (FBS). Cultures were grown for two days, and metaphase cells were arrested with colcemid after a 16-h incubation. Cells were then collected by trypsinization and centrifuged at $1000 \mathrm{rpm}$. Trypsin activity was inhibited by resuspension in medium containing 10\% FBS. The cells were pelleted by centrifugation, washed with phosphate-buffered saline, and resuspended in 
hypotonic medium $(0.075 \mathrm{M} \mathrm{KC} 1)$ for $20 \mathrm{~min}$ at $37^{\circ} \mathrm{C}$. Following hypotonic treatment, preparations were fixed three times with methanol:acetic acid (3:1).

The subsequent analysis was performed by GTG-banding and the results were interpreted according to the International System for Human Cytogenetic Nomenclature guidelines (Shaffer and Tommerup, 2005).

Twenty metaphase cells were examined. Chromosome banding analysis revealed the presence of a clonal translocation involving chromosomes 3 and 13. The karyotype was denoted as: 46,XY, t(3;13)(q21;q34) [2]/46,XY [18] (Figure 2D).

\section{DISCUSSION}

Malignant transformation of OC occurs mainly in the cartilaginous cap into low-grade chondrosarcomas. This phenomenon is believed to occur stepwise: "Osteochondroma giving rise to peripheral low-grade chondrosarcoma that in turn dedifferentiates into a high-grade sarcoma that may appear as fibrosarcoma, malignant fibrous histiocytoma, and osteosarcoma" (Garrison et al., 1982). Low-grade chondrosarcomas tend to grow in the periphery, enlarging the cartilaginous cap, which produces the clinical alert signs and can be confirmed in MRI (Lamovec et al., 1999; Nezhad et al., 2008).

Some osteosarcomas secondary to OC corroborate the stepwise theory and show all kinds of neoplastic tissue, such as normal osteochondroma, low-grade chondrosarcoma and a higher grade osteosarcoma with a chondral component as described by Nojima et al. (1991). Others develop from the spongeous bone of the stalk and have no relationship with the cartilaginous cap. No thickening of the cap is observed and they have no neoplastic cartilaginous components. This kind of secondary osteosarcoma is described by us and others (Lamovec et al., 1999; Bovée et al., 2002; Meissner et al., 2006; Nezhad et al., 2008).

The present report describes the late occurrence of a secondary osteosarcoma derived from an OC. Chromosome banding analysis of the transformed bone tissue revealed a clonal karyotype denoted as 46,XY, t(3;13)(q21;q34) [2]/46,XY [18]. Osteochondromas often show normal cytogenetics with loss of heterozygosity (LOH) at the EXT1 and EXT2 loci as the most characteristic aberrations (Bovée et al., 1999). However, some cytogenetic alterations have been detected, suggesting a clonal neoplastic growth (Mertens et al., 1994). In contrast, osteosarcomas are often characterized by an array of sequential and well-orchestrated genetic changes, with complex and heterogeneous chromosomal abnormalities (Sandberg and Bridge, 2003). This situation raises some doubts as to the stepwise evolution theory and the genetic stability of the stalk cancellous bone.

The cytogenetic analysis of our sample revealed a clonal cytogenetic aberration. Although microsatellite analysis for LOH was not performed in our sample, a recent study showed evidence of mechanisms alternative to the inactivation of the EXT genes during OC onset and neoplastic progression (Zuntini et al., 2010). The 3q21 band has been shown to be a recurrent breakpoint in cartilaginous tumors; nonetheless, the genetic mechanisms underlying transformation into a low-grade osteosarcoma still remain undisclosed (Sjögren et al., 2004).

Osteosarcomas arising from $\mathrm{OC}$ have been described thirteen times in the literature, ten of them from solitary OC. No clear alert sign in the prior resected OC could be detected at the histologic level to predict malignization in our patient. The genetic and molecular events associated with malignant transformation in this setting are still very poorly defined. Hope- 
fully, pooled genetic information on these cases may provide new insights into tumorigenesis and malignant transformation of OCs.

\section{ACKNOWLEDGMENTS}

Research supported by FAPESP (São Paulo State Research Foundation (\#07/54236-4 and \#06/04827-3).

\section{REFERENCES}

Bovée JV, Cleton-Jansen AM, Wuyts W, Caethoven G, et al. (1999). EXT-mutation analysis and loss of heterozygosity in sporadic and hereditary osteochondromas and secondary chondrosarcomas. Am. J. Hum. Genet. 65: 689-698.

Bovée JV, Sakkers RJ, Geirnaerdt MJ, Taminiau AH, et al. (2002). Intermediate grade osteosarcoma and chondrosarcoma arising in an osteochondroma. A case report of a patient with hereditary multiple exostoses. J. Clin. Pathol. 55: 226229.

Garrison RC, Unni KK, McLeod RA, Pritchard DJ, et al. (1982). Chondrosarcoma arising in osteochondroma. Cancer 49: 1890-1897.

Khurana J, Abdul-Karim F and Bovée JVMG (2002). Osteochondroma. In: World Health Organization Classification of Tumours. Pathology and Genetics of Tumours of Soft Tissue and Bone (Fletcher CDM, Unni KK and Mertens F, eds.). IARC Press, Lyon, 234-236.

Lamovec J, Spiler M and Jevtic V (1999). Osteosarcoma arising in a solitary osteochondroma of the fibula. Arch. Pathol. Lab. Med. 123: 832-834.

Lee KC, Davies AM and Cassar-Pullicino VN (2002). Imaging the complications of osteochondromas. Clin. Radiol. 57: $18-28$.

Meissner SA, Vieth V, August C and Winkelmann W (2006). Radiology-pathology conference: osteosarcoma in a cartilaginous exostosis of the femur. Clin. Imaging 30: 206-209.

Mertens F, Rydholm A, Kreicbergs A, Willen H, et al. (1994). Loss of chromosome band 8q24 in sporadic osteocartilaginous exostoses. Genes Chromosomes Cancer 9: 8-12.

Nezhad ST, Bagheri MH and Rakhshandero SH (2008). Osteosarcoma arising from a solitary osteochondroma of the tibia. IRCMJ 10: 341-343.

Nishida Y, Isu K, Ueda T, Nishimoto Y, et al. (2009). Osteosarcoma in the elderly over 60 years: a multicenter study by the Japanese Musculoskeletal Oncology Group. J. Surg. Oncol. 100: 48-54.

Nojima T, Yamashiro K, Fujita M, Isu K, et al. (1991). A case of osteosarcoma arising in a solitary osteochondroma. Acta Orthop. Scand. 62: 290-292.

Sandberg AA and Bridge JA (2003). Updates on the cytogenetics and molecular genetics of bone and soft tissue tumors: osteosarcoma and related tumors. Cancer Genet. Cytogenet. 145: 1-30.

Shaffer LG and Tommerup N (2005). ISCN 2005: An International System for Human Cytogenetic Nomenclature. Karger, Basel.

Sjögren H, Orndal C, Tingby O, Meis-Kindblom JM, et al. (2004). Cytogenetic and spectral karyotype analyses of benign and malignant cartilage tumours. Int. J. Oncol. 24: 1385-1391.

Staals EL, Bacchini P, Mercuri M and Bertoni F (2007). Dedifferentiated chondrosarcomas arising in preexisting osteochondromas. J. Bone Joint. Surg. Am. 89: 987-993.

Zuntini M, Pedrini E, Parra A, Sgariglia F, et al. (2010). Genetic models of osteochondroma onset and neoplastic progression: evidence for mechanisms alternative to EXT genes inactivation. Oncogene 29: 3827-3834. 